\title{
SELF-REPORTED SKIN CONCERNS: AN EPIDEMIOLOGICAL STUDY OF COMMUNITY- DWELLING OLDER PEOPLE
}

Cowdell, F., Dyson, J., Long, J., \& Macleod, U.

\section{ABSTRACT}

\section{Background}

Globally the population is getting older and it is essential to develop effective interventions to promote healthy ageing. Skin change with age is inevitable and renders this often neglected organ more vulnerable to damage and breakdown; this can be costly to individuals and society. Maintenance of skin health in older people presents a health challenge that has yet to be fully understood or addressed.

\section{Aim}

To identify the frequency and impact of self-reported skin concerns in community-dwelling older people.

\section{Methods}

Cross sectional, self-reported questionnaire survey in England. Patients registered with participating general practices $(n=3)$, aged $\geq 70$ years, living in their own homes and able to give informed consent $(n=3359)$ were sent a letter of invitation to a free health and care assessment and 1116 responded.

\section{Methods}

Data were collected from August 2015 - January 2016. An adapted version of the validated EASYCare Standard 2010 questionnaire was completed by self-report.

\section{Results}

When asked "do you have any concerns about your skin?" $16.5 \%(n=183)$ said yes. Of this group the most common concerns were dry skin $80.7 \%(n=146)$, itching $56.9 \%(n=103)$ and aged appearance $61 \%(n=113)$. Itch, dry skin and inflammation were rated as most bothersome. There was a significant association between the dry skin and itch $\chi^{2}(1)=6.9, p<0.05$.

This is the peer reviewed version of the following article: Cowdell F, Dyson J, Long J, Macleod U. Selfreported skin concerns: An epidemiological study of community-dwelling older people. Int J Older People Nurs. 2018;13:e12195, which has been published in final form at https://doi.org/10.1111/ opn.12195. This article may be used for non-commercial purposes in accordance with Wiley Terms and Conditions for Use of Self-Archived Versions. 1 
IJOPN 15.6.17 revised 31.10.17

\section{Conclusion}

Many community-dwelling older people suffer with skin concerns predominantly dry skin and itching that is often bothersome.

Implications for practice

Skin health assessment is often absent in routine consultations with community-dwelling older people. Dry, itchy skin is prevalent and can be simply managed with low-cost interventions. This has the potential to reduce suffering and maintain or improve skin barrier function. Nurses and other health professionals should therefore routinely assess and advise on skin health care for this population. 


\section{SUMMARY STATEMENT OF IMPLICATIONS FOR PRACTICE}

\section{What does this research add to existing knowledge in gerontology?}

- This is the first English and globally largest survey of skin health in community-dwelling older people

- The survey highlights the high prevalence of dry skin, itching and concerns about ageing appearance in community dwelling older people.

What are the implications of this knowledge for nursing care with older people?

- The high prevalence of dry skin and itching suggest that these conditions should be more routinely addressed in assessment.

- As dry skin and itching are amenable to simple, low-cost intervention routine assessment and resulting intervention advice could be effective for reducing suffering and improving skin barrier function.

How could findings be used to influence policy or practice or research or education?

- The study indicates that many community-dwelling older people suffer with bothersome dry and itchy skin, the need for routine assessment should be addressed in nursing education.

- There is a need to develop interventions to support community-dwelling older people with dry, itchy skin to self-manage these conditions as effectively as possible.

Keywords: community dwelling, epidemiology, nursing, older people, self-report, skin health 


\section{INTRODUCTION}

It is estimated that by 2050 there will be over 1.5 billion people aged $\geq 65$ years worldwide (Pew Research Centre, 2014). This demographic shift is positive in many respects; however, it represents a significant socio-economic challenge and demands that we re-think the way in which we approach healthy ageing. The increasing financial pressure on health and social care means that services are restricted to those with the highest needs; it is therefore imperative that there is a system for lowlevel support and advice to help older people remain independent and in their own homes for as long as possible (Wrosch et al., 2013). One element that is conspicuously absent from the healthy ageing agenda is consideration of maintenance and promotion of healthy skin; indeed healthy skin ageing has been identified as a "grand challenge" (Blume-Peytavi, et al., 2016). The skin is an important but often forgotten organ which acts as a protective barrier between the internal organs and the external environment and has sensory, thermoregulatory and vitamin D synthesis functions (Kottner, 2015). The appearance of the skin influences psychological wellbeing (Sampogna, et al., 2016) and skin breakdown can have a devastating impact on the individual and be costly to health and care services (Cowdell, \& Steventon, 2015). Maintenance of skin health is one of the cornerstones of nursing practice (Cowdell 2011).

\section{BACKGROUND}

As the skin ages it undergoes a range of intrinsic and extrinsic degenerative changes (Cowdell, 2010). Other factors that influence skin health in older age include "frequent washing, particularly with harsh products; lack of hygiene (producing a build-up of potential pathogens and an increased risk of infection); trauma; reduced peripheral sensation; reduced mobility; incontinence; depression and dementia; poly-pharmacy (taking multiple medications); diabetes and vascular changes; and poor nutrition" (Cowdell, et al., 2014). The combination of some or all of these risk factors can lead to impairment of skin barrier function which can make the skin significantly more vulnerable to damage and increase the risk of breakdown (Kottner, \& Surber., 2016). 
Despite the inescapability of skin ageing, remarkably little is known about the epidemiology of skin health in community-dwelling people older people. In a recent systematic review of 74 studies most prevalence and incidence data were identified for hospital or long-term care settings; it is not clear if any of the data were self-reported. The four most prevalent skin conditions were xerosis (5.4$85.5 \%)$, fungal infections $(14.3-64 \%)$, dermatitis $(1-58.7 \%)$, and benign skin tumours $(1.7-74.5 \%)$ (Hahnel, et al., 2016). This review identifies methodological weaknesses in many of the included studies which limit their value. Anecdotally many older people experience xerosis (skin dryness), pruritus (itching) and fissures (cracks) (Cowdell, et al., 2014) however robust data is scarce (Hahnel, et al., 2016). Only four epidemiological studies published in the last three decades have investigated prevalence in community-dwelling older people. Estimates of older people reporting any skin concern range from 13-83\% (Beauregard, \& Gilchrest., 1987; Fleischer, et al., 1996; Gontijo, et al. 2014; Paul, et al. 2011) with xerosis and pruritus being the most frequent. However the differing methodologies and populations of these studies limit their value in understanding the nature, frequency and impact of these concerns. At present there is limited evidence of routine assessment of skin health in consultations with community-dwelling older people.

Comprehensive health and social care assessment can be a useful guide to the needs of the older person. The EASYCare assessment instrument was developed, originally in a Consensus Conference sponsored by the World Health Organisation (Philp, 1997). EASYCare is based on a combination of existing validated scales for measuring health and care needs for the older person, for example the Medical Outcomes Survey Short Form 36 (Ware, \& Sherbourne., 1992) and the Barthel Index (Mahoney, \& Barthel., 1965). Whilst these measures are not perfect they are widely used and quick and simple to complete (Philp, 1997). A systematic review of EASYCare (Craig, et al., 2015) concludes from the 29 included papers that there is good evidence of validity and that acceptability, defined as ease of use in practice, is high but that reliability is yet to be proven. To date the 
instrument has been used in numerous settings and has been completed by health care practitioners and older people. It is suggested that completion by nurses is preferable to self-completion as selfreport may increase risk over or under-reporting (Craig, et al., 2015). EASYCare is written in clear and simple language which encourages systematic, holistic assessment using a conversational approach. It contains a structured set of 49 questions to help identify health and care needs which pose significant threats to independence and health and well-being across seven key domains comprising: seeing, hearing and communicating; looking after yourself; getting around; your safety; accommodation and finance; staying healthy and mental health and well-being? Importantly all questions are answered according to the perspective of the older person.

The EASYCare assessment can be completed with or without support. On completion needs of the older person are identified and a response is mobilised according to what is most important to them. Until recently the EASYCare paid little attention to skin health but this dimension has recently been added and is the focus of this paper. The new skin-focused item set was developed by extensive communication with a team of dermatology experts and were congruent with existing knowledge suggesting a degree of face validity.

\section{METHOD}

\section{Aim}

To identify the frequency and impact of self-reported skin concerns amongst a cohort of communitydwelling older people.

\section{Design}

Multi-centre, cross sectional, self-reported questionnaire study.

\section{Participants and procedure}

A letter of invitation to a free health and care assessment, with reply slip, was sent to all eligible patients registered with three General Practices in one town in north-east England $(n=3359)$. 
Inclusion criteria were patients aged $\geq 70$ years, living in their own homes and able to give informed consent. Exclusion criteria were patients who lived in a care home setting or who were unable to consent to take part. If they responded positively participants were contacted by a trained assessor within 21 days. One follow-up letter was sent to non-responders. Participants were given the option of a telephone or a face-to-face interview where they were asked the questions on the EASYcare Standard 2010. Telephone calls were conducted by contact centre assessors from a local telecommunications company who had pre-existing skills in out-bound calls and simply recorded responses. Calls were recorded and randomly monitored for quality throughout the project by a centre team leader. No quality issues were identified during random monitoring of call. Assessors had to develop skills in managing the call handling time as occasionally conversations had the potential to go beyond the EASYCare remit. Recordings were reviewed in instances where assessors had any concerns about the wellbeing of participants and required action was taken. Face-to-face meetings were undertaken by research trained assessors with social care or voluntary work experience; the focus in these meetings remained on self-reported skin concerns. All assessors attended a one day training programme in 'person-centred care' which comprised completion of a work-book (available from authors on request) with group discussion and activities to familiarise them with the instrument and how to conduct assessment.

\section{Data collection}

Data were collected between August 2015 and January 2016 using the EASYCare Standard 2010 questionnaire (EASYCare Group, 2014) with the addition of the item set regarding skin health. This amended questionnaire has not yet been published.

\section{FIGURE 1 HERE}

\section{Ethical considerations}

Ethical approval was granted by a Hull York Medical School Research Ethics Committee (ref 1507). 
NHS approval was not required as the research team were given access only to anonymised patient data.

\section{Data analysis}

Likert style data were entered into SPSS V23 (IBM, 2014) and analysed, in the main, descriptively. To establish the association between dry skin and itch, ulcers, inflammation and aged appearance chisquare test was used. Where an association was established odds ratio was calculated to establish the strength of the relationship.

\section{RESULTS}

Invitations were sent to 3359 patients and 1116 responded (a response rate of $33.2 \%$ ). Of the 1116 participants 873 completed the EASYCare instrument via the telephone with an assessor. Face-toface meetings were preferred by 243 patients largely due to hearing loss or having multiple issues to discuss. Of these $55.5 \%$ were women. The age range was 75 to 99 years (mean age 81.14 (SD 4.7)). When asked "do you have any concerns about your skin?" $16.5 \%$ of the $1116(n=183)$ said yes. If a person answered no at this stage no further skin health questions were asked. For an affirmative answer the person was asked to identify the nature of their concern. The results of this are summarised in Tables 1 and 2 according to the whole sample and those saying "yes" to a skin concern respectively.

\section{TABLE 1 HERE}

TABLE 2 HERE

Individuals who reported a concern were asked to rate the extent to which they found it bothersome on a scale of 1 to 4 with $1=$ not bothersome, 2 = a little bit bothersome, $3=$ a lot bothersome and $4=$ very much bothersome. Table 3 demonstrates the proportion of those reporting a concern that found it bothersome (as rated a little bit, a lot or very bothersome) and Figure 2 indicates the mean bothersome score per concern. 


\section{TABLE 3 HERE}

\section{FIGURE 2 HERE}

Of $16.5 \%$ ( $n=183$ ) who responded that they had a skin concern dry skin, aged appearance and itching were the most frequently reported. Dry skin was an issue for $80.7 \%(n=146), 61.7 \%(n=113)$ experienced an aged appearance and $56.9 \%(n=103)$ suffered with itching. The most bothersome conditions were itch (a little $46.4 \%$, a lot $27.7 \%$ and very much $5.4 \%$ (mean rating=2.18)), dry skin (a little $58.8 \%$, a lot $11.5 \%$ and very much $4.1 \%$ (mean rating=1.94)) and inflammation (a little $44.8 \%$, a lot $27.7 \%$ and very much $1.5 \%$ (mean rating=1.94)).

With regards to associations, there was no association between dry skin and ulcers or between dry skin and inflammation. There was a significant association between the dry skin and itch $\chi^{2}(1)=6.9$, $p<0.05$. This seems to represent the fact that based on the odds ratio people with dry skin were more likely to feel itch than those without dry skin by a factor of 2.6. There was a significant association between dry skin and aged appearance $\chi^{2}(1)=5.17, p<0.05$. This seems to represent the fact that based on the odds ratio people with dry skin were more likely to report their skin has an aged appearance compared with those without dry skin by a factor of 2.47 .

We considered any differences between telephone and face-to-face data collection. According to Chi squared test there was no significant difference between these two modes of communication for all but one item of skin health questions. The one item where a difference was identified was "you have aged skin appearance with wrinkles and brown spots on your skin" with people on the telephone more likely to say yes $(P<0.05)$.

\section{DISCUSSION}

This study demonstrates that a substantial number of community-dwelling older people have 
concerns about their skin health predominantly dry skin, itching and aged appearance which are bothersome to a greater or lesser degree. There was no association between dry skin and ulceration or between dry skin and inflammation. There is a significant association between dry skin and itching and between dry skin and aged appearance. The implication of this is that active management of dry skin may reduce itch and the associated risk of skin damage and help promote the look good, feel good' factor (Ong, \& Ryan., undated) which is important for wellbeing in older people (Penzer, \& Finch, 2001).

This study is one of very few that has investigated self-reported skin concerns in a large cohort of community-dwelling older adults. The sample size is the largest in published studies to date. It provided older people with an opportunity to define and report concerns from their own perspective in contrast to other studies which have offered a limited choice of pre-defined conditions (for example Gontijo, et al., 2014). This study provides new insights into these concerns that can be used to inform future interventions to support for maintenance and improvement of skin health. The response rate of $1116 / 3359(33 \%)$ is consistent with what other authors have found. A systematic review by Edwards, et al., (2005) includes papers reporting response rates of between one in ten (Newby, et al., 2003) to one in two (Gibson, et al., 1999). However most included papers found one in four (e.g. Whiteman, et al., 2003).

Limitations of the study include the new skin-focused item set was not validated as part of EASYCare Standard 2010. The survey was conducted in only one area of England. Factors such as exposure to ultraviolet radiation, dry or cool environmental air contribute to skin ageing and associated concerns (Farage et al., 2013, Baumann et al., 2009) it is therefore likely then that the results presented here are generalisable only so far as those from similar climates to the North of England. 
There is a risk that skin concerns may have been under-reported. The skin item set was not subject to validity testing, other than face validity, and was not considered in any reviews of the EASYCare. The screening skin question was just one among 49 core questions and if a negative answer was given to this no further enquiry was made regarding skin health. More probing questions may have revealed more concerns but for the sake of study replication we adopted the EASYCare approach with fidelity. Whilst a practitioner assessment of skin may have identified more skin "disease" there is evidence that self-report is an accurate measure of skin "condition" (e.g. Gontijo et al., 2014) which was the focus of this study.

Our study is broadly congruent with the findings of others. Four existing epidemiological studies examined skin health problems in the target population. Each had a different methodology and population precluding meta-analysis. However, results suggest a high prevalence of skin complaints. In 68 people aged 50 to 91 years, $66 \%$ of the whole group reported skin problems, rising to $83 \%$ for octogenarians with the most common disorder being pruritus (Beauregard, \& Gilchrest., 1987). Similarly Fleischer, et al., (1996) reports that of 204 people aged $>64$ years, 70\% experienced pruritus in the previous week with $34 \%$ stating that this symptom could not be ignored; $64 \%$ described a nonitching skin condition. Xerosis was the focus of a study of 756 patients aged $>65$ in primary care, $56 \%$ reported some degree of xerosis and of those reporting moderate to severe xerosis using the Overall Dry Skin Score (Serup, 1995) many experienced pruritus and very dry or unbearably dry skin. Community-dwelling adults aged over 65 years $(n=1985)$ completed a 16-item checklist of chronic medical conditions during two face-to-face interviews 12 months apart; $13.3 \%$ self-reported skin conditions at the first interview and $13.1 \%$ at second interview (Gontijo, et al., 2014). A drop down list of specific skin conditions (Gontijo, et al., 2014) did not allow for reporting of more general skin concerns which may account for the much lower prevalence in this study. 
IJOPN 15.6.17 revised 31.10.17

Instruments used to assess self-reported skin condition varied from 31-item and 33-item questionnaires administered by the examining physician (Fleischer, et al., 1996; Beauregard, \& Gilchrest., 1987). Questionnaires used are not included in the publications and attempts to obtain them have been unsuccessful; the validity and reliability is unknown. Paul, et al. (2011) reports that patients' personal assessment of skin dryness and pruritus was assessed, there is no clear information about how this was accomplished but mention is made of the Overall Dry Skin (ODS) (Serup, 1995) which includes a patient version using a visual analogue scale.

In contrast to the studies with multiple skin related questions Gontijo, et al., (2014) asked just one question specifically about skin disease as opposed to the broader concept of skin concerns (Gontijo, et al., 2014). Clinician skin examination in these studies revealed relatively low agreement between clinician and patient assessment of skin condition and disease. For example Beauregard \& Gilchrist (1987) report that many participants used inappropriate medical terms for skin conditions and some reported no complaints when a disease was clearly present. Similarly Fleischer, et al., (1996) identified a number of undiagnosed skin diseases and tumours. Significant association between patient report of xerosis and clinician ODS score is reported by Gontijo, et al., (2014). The available literature (Beauregard \& Gilchrist 1987; Fleischer, et al., 1996; Gontijo, et al., 2014; Paul, et al., 2011) suggests that self-report may be an accurate measure of skin condition but not as accurate in terms of skin disease.

There is a growing body of evidence that low level skin concerns such as xerosis and pruritus are common in the ageing population. It is noted that such conditions are often considered 'minor', they frequently go untreated (Kirkup, 2008) despite causing discomfort and actual or potential impairment of skin barrier function with increased risk of skin breakdown (Hahnel, et al., 2016). Skin health is one of the cornerstones of nursing practice (Cowdell, et al., 2014) and as such we need to take every opportunity to maintain and promote skin integrity particularly in the older population. At 
IJOPN 15.6.17 revised 31.10.17

present most research and practice efforts are directed towards management of incontinence associated dermatitis and leg and pressure ulceration. This work is vital, but of equal importance is the maintenance of skin health in the wider 'well' older population. Investment in simple, low-cost interventions (Brooks, et al., 2017) may minimise unnecessary discomfort and reduce the risk of future skin breakdown.

\section{CONCLUSION}

To date there is little empirical data on the prevalence of self-reported skin concerns in communitydwelling older people. In order to plan and implement interventions to promote and enhance skin health and integrity we first need to understand the nature of the problem. This study has contributed to this understanding.

\section{RELEVANCE TO CLINICAL PRACTICE}

Healthy ageing is the responsibility of all nurses providing care to the older person. To date little attention has been paid to healthy skin ageing, our study demonstrates that skin concerns are frequent. Seemingly 'minor' conditions are often bothersome and existing evidence suggests they often go untreated. Nurses are ideally placed to provide advice about maintaining and promoting skin health and alleviating these unnecessary discomforts. At present, in the absence of an empirical evidence base, guidance is based on best practice and there is a need for further research to identify optimum, cost-effective, evidence-based skin care regimens. 


\section{REFERENCES}

Beauregard, S., \& Gilchrest, B. (1987). A survey of skin problems and skin care regimens in the elderly. Archives of Dermatology, 123(12), 1638-1643.

Blume-Peytavi, U., Kottner, J., Sterry, W., Hodin, MW., Griffiths, T., Watson, R., Hay, RJ., \& Griffiths, C. (2016). Age-associated skin conditions and diseases: current perspectives and future options. The Gerontologist, 56 (Suppl 2) S230-S242.

Brooks, J., Cowdell, F., Ersser, S., \& Matts, P. (2017). Skin cleansing and emolliating for older people: A quasi-experimental pilot study. International Journal of Older People Nursing DOI: 10.1111/opn.12145

Cowdell, F. (2010). Promoting skin health in older people. Nursing Older People, 22(10), 21-26.

Cowdell, F., 2011. Older people, personal hygiene, and skin care. Medsurg Nursing, 20(5), p.235.

Cowdell, F., Jadotte, YT., Ersser, SJ. et al. , Danby, S., Walton, S., Lawton, S., Roberts, A., Gardiner, E., Ware, F., \& Cork, M. (2014). Hygiene and emollient interventions for maintaining skin integrity in older people in hospital and residential care settings (Protocol). Cochrane Database of Systematic Reviews 2014, Issue 12. Art. No.: CD011377. DOI: 10.1002/14651858.CD011377.

Cowdell, F., \& Steventon, K. (2015). Skin cleansing practices for older people: a systematic review. International Journal of Older People Nursing, 10(1), 3-13. 
Craig, C., Chadborn, N., Sands G., Tuomainen, H., \& Gladman, J. (2015). Systematic review of EASYcare needs assessment for community-dwelling older people. Age \& Ageing, 44(4), 559-565.

EASYCare Group (2014). EASYCare tool https://www.easycarehealth.co.uk/ (accessed 15 June 2017).

Edwards, P., Cooper, R., Roberts I \& Frost C (2005). Meta-analysis of randomised trials of monetary incentives and response to mailed questionnaires. British Medical Journal, 59, 987.

Fleischer, A., McFarlane, M., Hinds, M., \& Mittelmark, M. (1996). Skin conditions and symptoms are common in the elderly: the prevalence of skin symptoms and conditions in an elderly population. The Journal of Geriatric Dermatology, 4(3),78-87

Gibson, P., Koepsell, T., Diehr, P., \& Hale, C. (1999). Increasing response rates for mailed surveys of medicaid clients and other low-income populations. American Journal of Epidemiology, 149, 1057.

Gontijo, G.S., Vasiliadis, H., Préville, M., \& Berbiche, D. (2014). Skin conditions in community-living older adults: Prevalence and characteristics of medical care service use. Journal of Cutaneous Medicine and Surgery, 18(3), 186-194.

Hahnel, E., Lichterfeld, A., Blume-Peytavi, U., \& Kottner, J. (2016). The epidemiology of skin conditions in the aged: A systematic review. Journal of Tissue Viability http://ac.elscdn.com/S0965206X16300225/1-s2.0-S0965206X16300225-main.pdf? tid=bab9cb1e-ab3b-11e6b3a9-00000aab0f27\&acdnat=1479218550 f62ac5895e91a51296b31aa7602bf450 (accessed 15 June 2017)

IBM Corp, (2014). IBM SPSS Statistics for Windows, Version 23.0. Armonk, NY: IBM Corp.

Kirkup, M. (2008). Itching in elderly people Geriatric Medicine, 38 (12), 671-674

Kottner, J. (2015). Of Youth and Age - What are the Differences Regarding Skin Structure and Function? EWMA Journal, 15(2), 11-13.

Kottner, J., \& Surber, C. (2016). Skin care in nursing: A critical discussion of nursing practice and research. International Journal of Nursing Studies, 61, 20-28.

Mahoney, F.I., \& Barthel, D.W., (1965). Functional evaluation: The Barthel Index. Meryland State Medical Journal, 14, 61-65.

Menichetti, J., \& Graffigna, G. (2016). How older citizens engage in their health promotion: a qualitative research-driven taxonomy of experiences and meanings. BMJ Open, 6(7), e010402e010402.

Newby, R., Watson, J., \& Woodliff, D. (2003). SME survey methodology: response rates, data quality, and cost effectiveness. Entrepreneurship Theory and Practice, 28, 163-172.

Ong, C-K., \& Ryan, T. (undated). Healthy skin for all: a multi-faceted approach. Oxford. International Foundation for Dermatology.

Paul, C., Maumus-Robert, S., Mazereeuw-Hautier, J., Guyen, C., Saudez, X., \& Schmitt, A. (2011). Prevalence and Risk Factors for Xerosis in the Elderly: A Cross-Sectional Epidemiological Study in Primary Care. Dermatology, 223,260-265 
Penzer, R., \& Finch, M. (2001). Promoting healthy skin in older people. Nursing Older People, 13(8), 22-8.

Pew Research Centre (2014). Attitudes about Aging: A Global Perspective. Available from http://www.pewglobal.org/2014/01/30/attitudes-about-aging-a-global-perspective/ (accessed 15 June 2017)

Philp, I. (1997). Can a medical and social assessment be combined? Journal of the Royal Society of Medicine (Supplement), 90(32), 11-13.

Ware, J.E., \& Sherbourne, C.D. (1992). The MOS 36-item Short-Form Health Survey (SF-36): I. Conceptual framework and item selection. Medical Care, 30, 473-83.

Sampogna, F., Tabolli, S., Giannantoni, P., Paradisi, A., \& Abeni, D. (2016). Relationship Between Psychosocial Burden of Skin Conditions and Symptoms: Measuring the Attributable Fraction. Acta Dermato-Venereologica, 96(1), 60-63.

Serup, J. (1995) EEMCO guidance for the assessment of dry skin (xerosis) and ichthyosis: clinical scoring systems. Skin Research and Technology, 1, 109-114.

Whiteman, M., Langenberg, P., Kjerulff, K., Mccarter, R., \& Flaws, J. (2003). A randomized trial of incentives to improve response rates to a mailed women's health questionnaire. Journal of Women's Health, 12, 821-828.

Wrosch, C., Rueggeberg, R., \& Hopppmann, C.A. (2013). Satisfaction with social support in older adulthood: The influence of social support changes and goal adjustment capacities. Psychology \& Aging, 28(3), 875-885.

\section{TABLES AND FIGURES}

\section{Figure 1: Skin health item set}

\begin{tabular}{|l|l|}
\hline Question: & Answer options: \\
\hline Do you have any concerns about your skin (e.g. appearance, dry, & Yes/No \\
itchy, rash, skin ulcer, skin cancer, skin infection, other)? & \\
\hline$\bullet \quad$ If yes, what skin concerns do you have? & Yes/No \\
\hline Do you have dry skin? & Not at all/a little/a lot/very much \\
\hline$\bullet \quad$ If yes, how much does it bother you in your life? & Yes/No \\
\hline Do you have an itch? & \\
\hline
\end{tabular}




\begin{tabular}{|c|c|}
\hline - If yes, how much does it bother you in your life or sleep? & Not at all/a little/a lot/very much \\
\hline Do you have skin inflammation such as rash or eczema? & Yes/No \\
\hline - If yes, how much does it bother you in your life? & Not at all/a little/a lot/very much \\
\hline $\begin{array}{l}\text { Do you have skin ulcers such leg ulcers or pressure ulcers (bed } \\
\text { sores)? }\end{array}$ & Yes/No \\
\hline - If yes, how much does it bother you in your life? & Not at all/a little/a lot/very much \\
\hline Do you have (or had) skin pre-cancerous or cancerous lesions? & Yes/No \\
\hline - If yes, how much does it bother you in your life? & Not at all/a little/a lot/very much \\
\hline Do you have skin infection? & Yes/No \\
\hline - If yes, how much does it bother you in your life? & Not at all/a little/a lot/very much \\
\hline $\begin{array}{l}\text { Do you have aged skin appearance with wrinkles, brown spots, skin } \\
\text { laxity, skin thinning? }\end{array}$ & Yes/No \\
\hline $\begin{array}{l}\text { - If yes, how much does it bother you in your life or your } \\
\text { social interaction? }\end{array}$ & Not at all/a little/a lot/very much \\
\hline Do you have other skin problems? & Yes/No \\
\hline - If yes, please specify & Free text \\
\hline
\end{tabular}


IJOPN 15.6.17 revised 31.10.17

Figure 2: The degree to which people found their skin concern bothersome 
IJOPN 15.6.17 revised 31.10.17

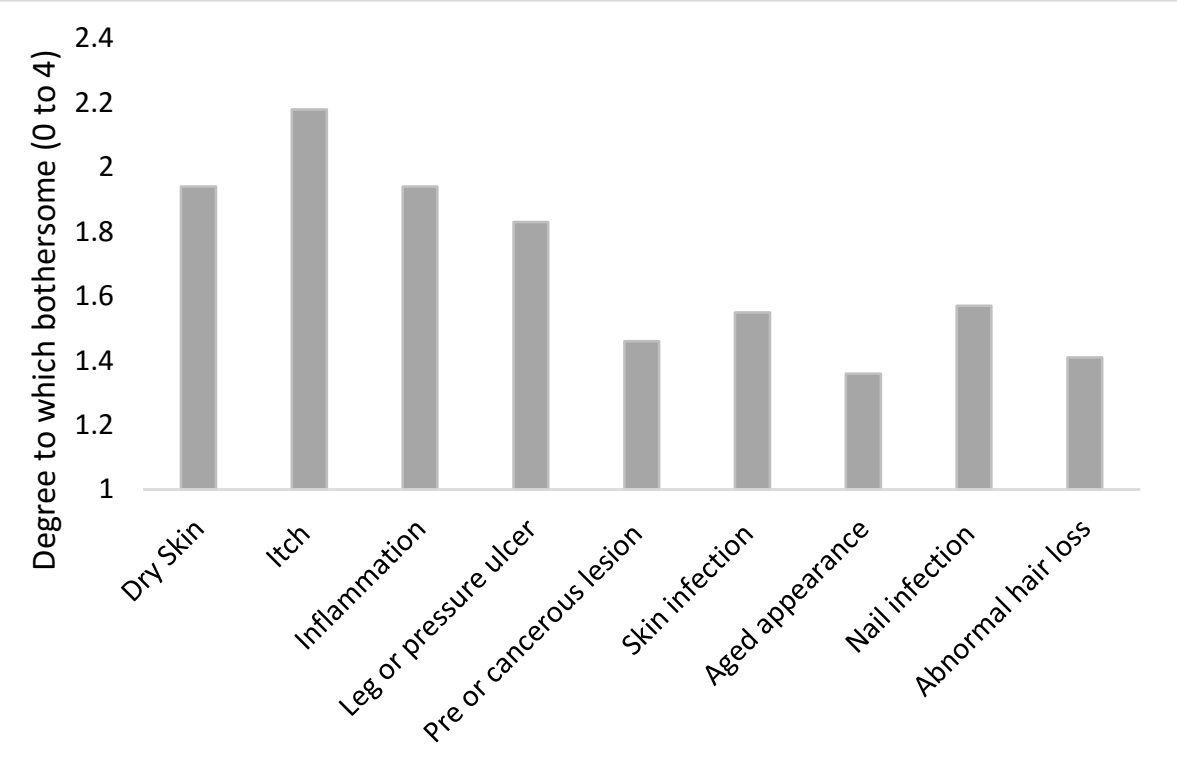

Table 1: The nature of the skin concern (all respondents) 
IJOPN 15.6.17 revised 31.10.17

\begin{tabular}{|l|l|}
\hline Concern & Percentage of all 1116 respondents (number) \\
\hline Dry Skin & $12.1(146)$ \\
\hline Itch & $9.3(104)$ \\
\hline Inflammation & $4.9(55)$ \\
\hline Leg or pressure ulcer & $1(11)$ \\
\hline Pre-cancerous or cancerous lesion & $1.5(17)$ \\
\hline Skin infection & $0.8(9)$ \\
\hline Aged appearance & $10.2(114)$ \\
\hline Nail infection & $2.2(25)$ \\
\hline Abnormal hair loss & $1(11)$ \\
\hline
\end{tabular}

Table 2: The nature of the skin concern (respondents that said "yes") 


\begin{tabular}{|l|l|}
\hline Concern & Percentage of those 183 that said "yes" to having \\
& a skin concern \\
\hline Dry Skin & $80.7(146)$ \\
\hline Itch & $56.9(103)$ \\
\hline Inflammation & $30.4(55)$ \\
\hline Leg or pressure ulcer & $6.1(11)$ \\
\hline Pre-cancerous or cancerous lesion & $8.7(16)$ \\
\hline Skin infection & $4.5(8)$ \\
\hline Aged appearance & $61(113)$ \\
\hline Nail infection & $13.3(24)$ \\
\hline Abnormal hair loss & $6.1(11)$ \\
\hline
\end{tabular}

Table 3: The nature of the problem for those identifying skin concerns and the degree to which these were bothersome 
IJOPN 15.6.17 revised 31.10.17

\begin{tabular}{|l|l|}
\hline Concern & $\begin{array}{l}\text { Proportion of people reporting the concern to be } \\
\text { a little, a lot or very bothersome (number) }\end{array}$ \\
\hline Dry Skin & $80.7(146)$ \\
\hline Itch & $56.9(103)$ \\
\hline Inflammation & $30.4(55)$ \\
\hline Leg or pressure ulcer & $6.1(11)$ \\
\hline Pre-cancerous or cancerous lesion & $8.7(16)$ \\
\hline Skin infection & $4.5(8)$ \\
\hline Aged appearance & $61(113)$ \\
\hline Nail infection & $13.3(24)$ \\
\hline Abnormal hair loss & $6.1(11)$ \\
\hline
\end{tabular}

